continued to show A. baumannii. Rifampicin was added. The patient was weaned to pressure support ventilation (PSV) trials. Later his chest infection worsened with signs of sepsis. He developed multiorgan failure with deranged liver and renal function test. He became comatosed, hemodynamically unstable, and needed high ventilatory support and hemodialysis. Finally, the patient died of sepsis and multiorgan failure after 33 days of treatment.

Conclusion: Though ECMO is now becoming the modality of choice for H1N1 pneumonia and severe ARDS, but complete aseptic precautions must be kept throughout the treatment to achieve successful outcome.

\section{A3 Case Series of Patients with Aluminium Phosphate Poisoning Treated with Extracorporeal Membrane Oxygenation in Unique Hospital, Surat, Gujarat Samir Gami, ${ }^{1}$ Dipak Viradia, ${ }^{1}$ Haresh Vastarpara, Khushbu Vaghasiya ${ }^{1}$ ${ }^{1}$ Department of Critical Care, Unique Hospital, Surat, Gujrat, India}

Study period is from September 12, 2016 to December 15,2017 . Our center has treated 19 patients with severe aluminum phosphate (AP) poisoning with ECMO in the given period. We treated 12 more patients with the same poisoning but with very mild effect. The patients were managed conservatively with inotrope and hemodialysis without ECMO. Choice of treatment given was based on severity of poisoning. Those patients who have severe left ventricular (LV) dysfunction, lactic acidosis, and hypotension were treated with extracorporeal membrane oxygenation (ECMO).

All 19 patients were put on venovenous ECMO (VA-ECMO) in view of severe myocardial dysfunction. Out of 19, 12 were male and 7 were female. Fourteen patients had consumed poison in tablet form (average 2.7 tablets) and the remaining patients had taken poison in powder form (60 g). As awareness has increased in the society and the referring physicians, patients started coming early to the hospital, with the average time to hospital at 220 minutes. The earliest patient reached at 90 minutes and the maximum delayed case arrived at 540 minutes. As the center was performing more and more ECMO, our initiation time from hospital to reach to "on pump" was reduced from 120 to 30 minutes, with the average of 60 minutes. All ECMOs were initiated with "femfem" route done by percutaneous technique by intensivist in 18 patients, whereas one was cannulated with open surgical technique.

Arterial cannulas in most of the cases are $16 \mathrm{~F}$ size whereas venous drainage cannulas are $25 \mathrm{~F}$ size. Distal perfusion cannula (size $7-11 \mathrm{~F}$, average $8 \mathrm{~F}$ ) was put in all except two patients.

On admission, average $\mathrm{pH}, \mathrm{HCO}_{3}$, and lactate were $7.2,9.8$, and 13 , respectively. All the patients had very poor LV function with an average of $17 \%$. After we put patients on
VA-ECMO, all parameters were improved in majority of patients. Average $\mathrm{pH}, \mathrm{HCO}_{3}$, and lactate after 24 hours of ECMO were $7.3,15$, and 11 , respectively, with improved LV function of $20 \%$ on average.

In view of severe academia and lactic acidosis, we started sustained low-efficiency dialysis (SLED) in all patients, and after 24 hours in patients who had acute kidney injury (AKI), we continued with hemodialysis. All patients were on continuous intravenous (IV) heparin to maintain activated clotting time (ACT) in between 160 and 180 . Two patients developed distal limb ischemia on ECMO, and we needed surgical intervention in both of them. One patient recovered very well with normal myocardium but developed severe cerebral edema, with absent brainstem activity, so the patient was declared brain-dead and ECMO was removed. Out of 19 patients, 2 developed gastrointestinal (GI) bleed that was managed conservatively. In one patient, arterial cannula was dislodged accidentally and emergency arterial repair was performed.

Conclusion: All patients who survived required ECMO support for 96 hours on an average (shortest run was 48 hours and longest was 384 hours). Eleven patients survived out of 19 put on ECMO. The patients who came early for treatment had better outcome as compared to late presenters. Patients with persistence high lactate and low ejection fraction (EF) after 24 hours had poor outcome. Distal limb ischemia and GI bleed are major concern for ECMO management. There are many new indications for ECMO use coming, and this is adding one more to the list.

A4 A Rare Case of Coronary Cameral Fistula with Patent Ductus Arteriosus, National Heart Institute, New Delhi Arvind Prakash, ${ }^{1}$ Rachita Dhawan, ${ }^{1}$ Anirban Kundu, ${ }^{2}$ Vikas Ahalawhat, ${ }^{2}$ Om Prakash Yadava ${ }^{2}$

${ }^{1}$ Department of Cardiac Anaesthesia, National Heart Institute, New Delhi, India

2Department of Cardiac Surgery, National Heart Institute, New Delhi, India

A 34-year-old male patient underwent transthoracic echocardiography for the investigation of breathlessness and atypical chest discomfort. Clinical examination was unremarkable. A standard 12-lead electrocardiogram (ECG) demonstrated left ventricular hypertrophy with anterior T-wave inversion. Transthoracic echocardiography demonstrated a normally functioning left ventricle with left coronary cameral fistula draining in the left atrium (LA) with a tiny patent ductus arteriosus (PDA). The cardiac catheterization revealed a large coronary cameral fistula with ramus intermedius branch draining into the LA. The patient was started on $\beta$-blockers and diuretics, and taken up for PDA ligation and closure of the coronary cameral fistula on cardiopulmonary bypass (CPB). The postoperative transesophageal echocardiography (TEE) revealed no abnormal flows in the fistulous chamber or LA. After 8 